\title{
A Novel Decentralized Fixed-Time Tracking Control for Modular Robot Manipulators: Theoretical and Experimental Verification
}

\section{Zengpeng Lu}

Changchun University of Technology

\section{Yuanchun Li}

Changchun University of Technology

Yan Li (Dly6655@126.com )

Changchun University of Technology

\section{Research Article}

Keywords: Fixed-time convergence, Modular robot manipulators, Disturbance observer, Lyapunov stability

Posted Date: December 28th, 2021

DOI: https://doi.org/10.21203/rs.3.rs-1147639/v1

License: (c) (i) This work is licensed under a Creative Commons Attribution 4.0 International License.

Read Full License 


\title{
A novel decentralized fixed-time tracking control for modular robot manipulators: theoretical and experimental verification
}

\author{
Zengpeng $\mathrm{Lu}^{\mathrm{a}}$, Yuanchun $\mathrm{Li}^{\mathrm{a}}$, Yan $\mathrm{Li}^{\mathrm{a}, *}$ \\ ${ }^{a}$ Department of Control Science and Engineering, Changchun University of Technology, \\ Changchun, China
}

\begin{abstract}
This paper proposes a novel decentralized fixed-time tracking control approach, which realizes the advantages of modular robot manipulators (MRMs) with fixed-time convergence, strong robustness, and high tracking performance. First, to estimate the total uncertainty of MRMs, the fixed-time disturbance observer based on the extended state is developed. Then, combined with the disturbance observer, a novel decentralized control method based on fixed-time control strategy was developed to achieve the global fixed-time convergence of MRMs. And, stability analysis based on Lyapunov is used to obtain the fixed-time stability and convergence time of MRMs. Finally, numerical analysis and experiment respectively verify the excellent tracking ability of the proposed decentralized fixed-time tracking control algorithm.
\end{abstract}

Keywords: Fixed-time convergence, Modular robot manipulators, Disturbance observer, Lyapunov stability

\section{Introduction}

Modular robot manipulators (MRMs) [1, 2], have attracted widespread attention in the robotics community because of their structural flexibility and

\footnotetext{
* Corresponding author

Email address: 1y6655@126.com (Yan Li)

$U R L$ : luzengpeng@outlook.com (Zengpeng Lu), liyc@ccut.edu.cn (Yuanchun Li)
}

Preprint submitted to Journal of ${ }^{A} T_{E} X$ Templates

December 16, 2021 
adaptability better than traditional robots. So far, MRMs have been widely used in many extreme situations, such as medical assistance, disaster investigation, disaster relief, and space exploration. Correspondingly, it is still challenging to design an appropriate control system to ensure that MRMs can operate with high precision, safety, and reliability. These challenges include model uncertainty, uncertain disturbances, and highly nonlinear systems.

The performance of a mechanical system can be judged by various technical indicators, including transient response, convergence speed, steady-state accuracy, and robustness. At present, there are many technologies to deal with uncertainty and disturbance, including sliding mode control (SMC), neural network, adaptive fuzzy control, etc. Among them, SMC is widely used as an excellent robust control method due to its mature performance in system uncertainty and disturbance rejection [3 6]. In [7], the hierarchical SMC based on backstepping is presented to realize the balance and movement of the ballbot with model uncertainty and disturbance. In [8], SMC based on a cascaded extended state observer is proposed for flexible joint robots with uncertainties and disturbances. Unfortunately, the conventional SMC usually uses a linear sliding mode surface to make the system state asymptotically converge to the equilibrium point, but its convergence time tends to be infinite. In order to improve efficiency in actual production, the operation time of the robot is only a short moment. This urgently requires the robot to converge quickly to ensure that MRMs stably reaches the target position before performing the operation task, so as to improve the operation accuracy. Therefore, the terminal sliding mode controller (TSMC) has been developed to allow the control system to converge within finite time [9] 13 . Different from the traditional SMC, the nonlinear sliding hyperplane is adopted in TSMC to ensure that the system state so achieves rapid finite time convergence in the sliding phase [14 17]. In literature [18, the accurate tracking of redundant manipulators with model uncertainty and external disturbance is achieved by combining fuzzy adaptive and TSMC. In [19, the design of a non-singular TSMC was introduced to solve the problem of finite time trajectory tracking of the manipulator robot with full-state con- 
straints. As mentioned in 14 19, TSMC not only has the characteristics of a convergent point within finite time, but also has other superior characteristics, including higher tracking accuracy, faster convergence, and so on. However, the finite time convergence speed depends on the initial value, which usually may not be predictable in advance. At the same time, this means that the larger the 40 initial value, the longer the convergence time will be.

To circumvent the drawbacks of convergence within finite time and at the same time as a development of finite time control, fixed-time SMC has been proposed recently [20. At present, fixed-time controllers have been successfully used to aircraft [21, 22], spacecraft [23] and motor systems [24], etc., and there 45 are also some results in robot control systems [25 27]. In 28, a robust controller was presented to solve the problem of uncertain robot manipulators tracking and ensure the global approximate fixed-time convergence of the robot system. Considering a robot manipulator system with input constraints, an adaptive fixed-time fault-tolerant control is presented in [29]. According to the abovementioned documents, the advantage of fixed-time SMC is that it can ensure that the system converges and stabilizes within a fixed time, and the settling time does not depend on the initial state value.

Moreover, in most practical systems, uncertainty and external interference are inevitable [30, 31]. To boost the performance of the control system, observerbased control algorithms are widely used through real-time accurate estimation and feedback [32 36]. Among them, due to the simplicity of the disturbance observer, it is very suitable for application in the actual system and will not increase the calculation problem of the system. Therefore, based on the idea of the extended state observer, all the unknown uncertainties affecting the system are extended to a new state to enhance the estimation speed. Unfortunately, the disturbance observer based on the extended state has not yet obtained or guaranteed the stability of the fixed-time. Therefore, this paper presented a fixed-time disturbance observer based on an extended state to estimate the uncertainties of MRMs.

Inspired by the above-mentioned literature, this paper combines the ex- 
tended state-based disturbance observer with fixed-time control to form a novel type of decentralized fixed-time SMC strategy based on the fixed-time disturbance observer. First, based on joint torque feedback technology (JTF), the dynamic model of MRMs is presented as a collection of dynamic subsystems related to all coupled modules. Then, the uncertainty of the system is integrated into the total disturbance, which is estimated by a fixed-time disturbance observer based on an extended state. Secondly, a novel decentralized control method based on a fixed-time SMC strategy is designed in conjunction with the disturbance observer to accomplish global fixed-time convergence of 75 MRMs. In summary, the main achievements and novelties can be highlighted as follows:

1. To our knowledge, this is the first time that a fixed-time SMC approach has been introduced to solve the decentralized control problem of MRMs with model uncertainties and uncertain disturbances based on joint torque feedback technology.

2. Different from the existing methods that do not consider dynamic decomposition, model uncertainty, and external disturbance compensation control, this paper presents a decentralized tracking control scheme based on a fixedtime disturbance observer, including uncertainty decomposition-based robust control and observer-based fixed-time SMC. The proposed observer can realize fast and accurate lumped disturbance estimation in a fixed time, avoiding the delay in transmitting uncertain information to the system. And the established experimental platform verifies the advantages of the proposed method.

The follow-up structure of this paper is as follows: Dynamic model and problem statement in Sect. 2. In Sect. 3, the decentralized controller is proposed and its stability is proved. Numerical simulations and experiments are performed to verify the effectiveness of the proposed strategy in Sect. 4. The conclusion is given in Sect. 5 . 


\section{Dynamic model and problem statement}

95

\subsection{Dynamic model formulation}

As in Fig, 1, the MRMs system under consideration consists of $n$ modules. Each module has a harmonic reducer and a torque sensor, which are integrated into the rotary joint. Similar to the literature [37, the following assumptions are made to apply to each joint module of MRMs:

100

Assumption 1: The flexibility of the joint is negligible.

Assumption 2: The rotor is symmetric concerning the rotation axis.

Assumption 3: The inertia between the torque sensor and the reducer can be ignored, and the torque transmission will not fail at the reducer.

The following notations are used for the $i^{t h}$ module:

105

$I_{m i}:$ the moment of inertia of the joint motor;

$\gamma_{i}$ : the reduction ratio $\left(\gamma_{i} \geq 1\right)$;

$q_{i}$ : the angular position of the joint module;

$f_{i}\left(q_{i}, \dot{q}_{i}\right)$ : jonit friction;

$\tau_{f i}$ : Coupling torque information obtained by joint torque sensor;

110

$\tau_{i}:$ DC motor output torque;

$z_{i}$ : unit vector along the rotary axis of joint $i$; and

$z_{m i}$ : unit vector along the rotary axis of the $i^{\text {th }}$ rotor.

The dynamic equations derived from [37, which represents the MRMs system with $n$ rotating joints and joint torque sensors.

First, $i=1$, the basic module is described as:

$$
I_{m 1} \gamma_{1} \ddot{q}_{1}+f_{1}\left(q_{1}, \dot{q}_{1}\right)+\frac{\tau_{f 1}}{\gamma_{1}}=\tau_{1}
$$

115

Next, $i=2$, adjacent module is described as:

$$
I_{m 2} \gamma_{2} \ddot{q}_{2}+f_{2}\left(q_{2}, \dot{q}_{2}\right)+I_{m 2} z_{m 2}^{T} z_{1} \ddot{q}_{1}+\frac{\tau_{f 2}}{\gamma_{2}}=\tau_{2}
$$




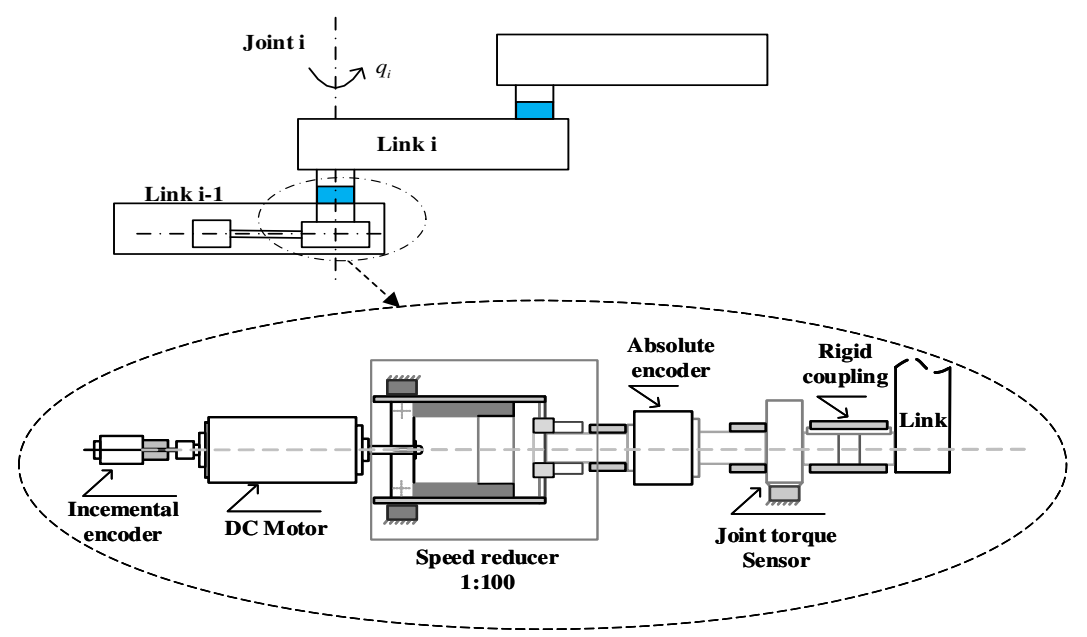

Figure 1: Structure diagram of the joint module.

For $i \geq 3$,

$$
\begin{aligned}
& I_{m i} \gamma_{i} \ddot{q}_{i}+f_{i}\left(q_{i}, \dot{q}_{i}\right)+I_{m i} \sum_{j=1}^{i-1} z_{m i}^{T} z_{j} \ddot{q}_{j}+\frac{\tau_{f i}}{\gamma_{i}} \\
&+I_{m i} \sum_{j=2}^{i-1} \sum_{k=1}^{j-1} z_{m i}^{T}\left(z_{k} \times z_{j}\right) \dot{q}_{k} \dot{q}_{j}=\tau_{i}
\end{aligned}
$$

where $f_{i}\left(q_{i}, \dot{q}_{i}\right)$ indicates the set of friction, which mainly includes the friction of each joint motor and reducer. Inspired by [38, 39], the expression for the friction term is as follows:

$$
f_{i}\left(q_{i}, \dot{q}_{i}\right)=B_{i} \dot{q}_{i}+F_{q i}\left(q_{i}, \dot{q}_{i}\right)+\left(F_{c i}+F_{s i} \exp \left(-F_{\tau i} \dot{q}_{i}^{2}\right)\right) \operatorname{sign}\left(\dot{q}_{i}\right)
$$

where $B_{i}, F_{c i}, F_{s i}, F_{\tau i}$ represents the coefficient of viscous friction, the related parameter of Coulomb friction, static friction related parameter, and the Stribeck effect parameter, respectively. $F_{q i}\left(q_{i}, \dot{q}_{i}\right)$ represents the non-parametric friction term that reflects the positional correlation of friction with other friction modeling errors. $\operatorname{sign}\left(\dot{q}_{i}\right)$ function is as follows:

$$
\operatorname{sign}\left(\dot{q}_{i}\right)=\left\{\begin{array}{cc}
1 & \text { for } \dot{q}_{i}>0 \\
0 & \text { for } \dot{q}_{i}=0 \\
-1 & \text { for } \dot{q}_{i}<0
\end{array}\right.
$$




\subsection{Model uncertainty analysis}

On the basis of Eq.(3), the dynamic model of MRMs with model uncertainty and uncertain disturbances can be written as:

$$
\begin{array}{r}
I_{m i} \gamma_{i} \ddot{q}_{i}+I_{m i} \sum_{j=2}^{i-1} \sum_{k=1}^{j-1} z_{m i}^{T}\left(z_{k} \times z_{j}\right) \dot{q}_{k} \dot{q}_{j}+I_{m i} \sum_{j=1}^{i-1} z_{m i}^{T} z_{j} \ddot{q}_{j} \\
+f_{i}\left(q_{i}, \dot{q}_{i}\right)+\frac{\tau_{f i}}{\gamma_{i}}+\tau_{d}=\tau_{i}
\end{array}
$$

where $\tau_{d}$ is the uncertain disturbance. The second term $I_{m i} \sum_{j=2}^{i-1} \sum_{k=1}^{j-1} z_{m i}^{T}\left(z_{k} \times z_{j}\right) \dot{q}_{k} \dot{q}_{j}$ and the third term $I_{m i} \sum_{j=1}^{i-1} z_{m i}^{T} z_{j} \ddot{q}_{j}$ of Eq. 60 are generated by the reconfiguration of MRMs. In order to make the equation more concise and compact, the following equation is given:

$$
\delta_{i}=\left\{\begin{array}{lr}
0 & i=1 \\
I_{m 2} z_{m 2}^{T} z_{1} \ddot{q}_{1} & i=2 \\
I_{m i} \sum_{j=1}^{i-1} z_{m i}^{T} z_{j} \ddot{q}_{j}+I_{m i} \sum_{j=2}^{i-1} \sum_{k=1}^{j-1} z_{m i}^{T}\left(z_{k} \times z_{j}\right) \dot{q}_{k} \dot{q}_{j} & i \geq 3
\end{array}\right.
$$

Joint friction is one of the important factors leading to the uncertainty of the model. By linearizing the friction (4) and combining it with Eq. (7), Eq. (6) can be rewritten as:

$$
\begin{aligned}
& I_{m i} \gamma_{i} \ddot{q}_{i}+\hat{B}_{i} \dot{q}_{i}+\left(\hat{F}_{c i}+\hat{F}_{s i} \exp \left(-\hat{F}_{\tau i} \dot{q}_{i}^{2}\right)\right) \operatorname{sign}\left(\dot{q}_{i}\right) \\
& \quad-Y\left(\dot{q}_{i}\right) \tilde{\theta}_{i}+F_{q i}\left(q_{i}, \dot{q}_{i}\right)+\delta_{i}+\frac{\tau_{f i}}{\gamma_{i}}+\tau_{d}=\tau_{i}
\end{aligned}
$$

where $Y\left(\dot{q}_{i}\right)=\left[\begin{array}{lll}\dot{q}_{i} & \operatorname{sign}\left(\dot{q}_{i}\right) \quad \exp \left(-\hat{F}_{\tau i} \dot{q}_{i}^{2}\right) \operatorname{sign}\left(\dot{q}_{i}\right) \quad-\hat{F}_{s i} \dot{q}_{i}^{2} \exp \left(-\hat{F}_{\tau i} \dot{q}_{i}^{2}\right) \operatorname{sign}\left(\dot{q}_{i}\right)\end{array}\right]$, and the parametric model uncertainty $\tilde{\theta}_{i}$ is defined as: $\tilde{\theta}_{i}=\left[\begin{array}{llll}\hat{B}_{i}-B_{i} & \hat{F}_{c i}-F_{c i} & \hat{F}_{s i}-F_{s i} & \hat{F}_{\tau i}-F_{\tau i}\end{array}\right] . \hat{F}_{\tau i}, \hat{B}_{i}, \hat{F}_{s i}$, and $\hat{F}_{c i}$ are the estimated parameters of the friction model, respectively.

Furthermore, MRMs have the following properties as they are used in the development of the proposed decentralized fixed-time tracking control.

Property 1: The vector products between $z_{m i}$ and $z_{i}$ are bounded as:

$$
\left|z_{m i}^{T} z_{j}\right| \leq 1, \quad\left|z_{m i}^{T}\left(z_{k} \times z_{j}\right)\right| \leq 1
$$


Property 2: The non-parametric friction term $F_{q i}\left(q_{i}, \dot{q}_{i}\right)$ is bounded as:

$$
\left|F_{q i}\left(q_{i}, \dot{q}_{i}\right)\right|<\rho_{i}
$$

Property 3: The subsystem uncertain disturbance is bounded as:

$$
\left|\tau_{d}\right|<\rho_{f i}
$$

\section{A novel decentralized fixed-time tracking controller desigen}

\subsection{Extended state based fixed-time disturbance observer}

Bsaed on the dynamic model (8), the extended state based fixed-time disturbance observer can be designed as:

$$
\left\{\begin{aligned}
& \dot{\hat{x}}_{i 2}= M_{i} \tau_{i}-M_{i}\left(\hat{B}_{i} \dot{q}_{i}+\left(\hat{F}_{c i}+\hat{F}_{s i} \exp \left(-\hat{F}_{\tau i} \dot{q}_{i}^{2}\right)\right) \operatorname{sign}\left(\dot{q}_{i}\right)\right)+F_{Q}\left(q_{i}, \dot{q}_{i}\right) \\
&-M_{i} \frac{\tau_{f i}}{\gamma_{i}}+M_{i} Y\left(\dot{q}_{i}\right) \widetilde{\theta}_{i}+\hat{v}_{i}+\alpha_{i} \llbracket e_{i} \rrbracket^{\mu_{i}}+\beta_{i} \llbracket e_{i} \rrbracket^{\nu_{i}} \\
& \dot{\hat{v}}_{i}=\varsigma_{i} \llbracket \varpi_{i} \rrbracket^{\varphi_{i}}+\xi_{i} \llbracket \varpi_{i} \rrbracket^{\psi_{i}}+L_{i} \operatorname{sign}\left(\varpi_{i}\right)
\end{aligned}\right.
$$

where $M_{i}=\left(I_{m i} \gamma_{i}\right)^{-1}, F_{Q}\left(q_{i}, \dot{q}_{i}\right)=-M_{i} F_{q i}\left(q_{i}, \dot{q}_{i}\right), \hat{v}=-M_{i} \delta_{i}(q, \dot{q}, \ddot{q})-M_{i} \tau_{d}$, $e_{i}=x_{i 2}-\hat{x}_{i 2}, \varsigma_{i}$ and $\xi_{i}$ stand for the positive constant, $\varphi_{i}>1,0<\psi_{i}<1$, and $L_{i}$ is a control gain.

$$
\begin{gathered}
\varpi_{i}=\dot{e}_{i}+\alpha_{i} \llbracket e_{i} \rrbracket^{\mu_{i}}+\beta_{i} \llbracket e_{i} \rrbracket^{\nu_{i}} \\
\dot{e}_{i}=\dot{x}_{i 2}-\dot{\hat{x}}_{i 2}=v_{i}-\hat{v}_{i}-\alpha_{i} \llbracket e_{i} \rrbracket^{\mu_{i}}-\beta_{i} \llbracket e_{i} \rrbracket^{\nu_{i}}
\end{gathered}
$$

Theorem 1: Considering the MRMs system (8), the extended state based fixedtime disturbance observer designed in 12 is employed to estimate the uncertainty term with $\left|\dot{v}_{i}\right| \leq L_{i}$. Then, the estimation error of the observer converges to zero within fixed-time.

The Lyapunov function $V_{i 1}=0.5 \sum_{i=1}^{n} \varpi_{i}^{2}$ is adopted, and its time derivative 
is calculated as:

$$
\begin{aligned}
\dot{V}_{i 1} & =\sum_{i=1}^{n} \varpi_{i} \dot{\varpi}_{i} \\
& =\sum_{i=1}^{n} \varpi_{i}\left(\dot{v}_{i}-\dot{\hat{v}}_{i}\right) \\
& =\sum_{i=1}^{n} \varpi_{i}\left(\dot{v}_{i}-\varsigma_{i} \llbracket \varpi_{i} \rrbracket^{\varphi_{i}}+\xi \llbracket \varpi_{i} \rrbracket^{\psi_{i}}+L_{i} \operatorname{sign}\left(\varpi_{i}\right)\right) \\
& \leq-\varsigma_{i} \sum_{i=1}^{n}\left|\varpi_{i}\right|^{\varphi_{i}+1}-\xi_{i} \sum_{i=1}^{n}\left|\varpi_{i}\right|^{\psi_{i}+1} \\
& \leq-2^{\frac{\varphi_{i}+1}{2}} n^{\frac{1-\varphi_{i}}{2}} \varsigma_{i}\left(V_{i 1}^{\frac{\varphi_{i}+1}{2}}\right)-2^{\frac{\psi_{i}+1}{2}} \xi_{i}\left(V_{i 1}^{\frac{\psi_{i}+1}{2}}\right)
\end{aligned}
$$

From (21),

$$
\begin{aligned}
& \dot{V}_{i 1} \leq-2^{\frac{\varphi_{i}+1}{2}} n^{\frac{1-\varphi_{i}}{2}} \varsigma_{i}\left(V_{i 1}^{\frac{\varphi_{i}+1}{2}}\right)-2^{\frac{\psi_{i}+1}{2}} \xi_{i}\left(V_{i 1}^{\frac{\psi_{i}+1}{2}}\right) \\
& \dot{V_{i 1}}+2^{\frac{\varphi_{i}+1}{2}} n^{\frac{1-\varphi_{i}}{2}} \varsigma_{i}\left(V_{i 1}^{\frac{\varphi_{i}+1}{2}}\right)+2^{\frac{\psi_{i}+1}{2}} \xi_{i}\left(V_{i 1}^{\frac{\psi_{i}+1}{2}}\right) \leq 0
\end{aligned}
$$

Under Lemma 1, the settling time $T_{0}$ is bounded as the following:

$$
T_{0} \leq \frac{1}{2^{\frac{\varphi_{i}+1}{2}} n^{\frac{1-\varphi_{i}}{2}} \varsigma_{i}\left(\frac{\varphi_{i}+1}{2}-1\right)}+\frac{1}{2^{\frac{\psi_{i}+1}{2}} \xi_{i}\left(1-\frac{\psi_{i}+1}{2}\right)}
$$

Therefore, the disturbance observer can be estimated the uncertainties in fixed-time.

Remark 1: In Eq. (12), the extended state gain $L_{i}$ is assumed with $\left|\dot{v}_{i}\right| \leq L_{i}$. To satisfy $\left|\dot{v}_{i}\right| \leq L_{i}, L_{i}$ is obtained through the two-layer adaptive rule given in [41,

$$
\left\{\begin{array}{l}
\dot{L}_{i}(t)=-\left(\rho_{i 0}+\rho_{i}\right) \operatorname{sign}\left(\delta_{i}(t)\right), \rho_{i 0}>0 \\
\dot{\rho}_{i}=\beta_{i 1}\left|\delta_{i}(t)\right|, \beta_{i 1}>0
\end{array}\right.
$$

where

$$
\begin{aligned}
& \delta_{i}=L_{i}(t)-\frac{\left|\kappa_{i}\right|}{\ell_{i 0}}-\ell_{i 1}, \ell_{i 0}, \ell_{i 1}>0 \\
& \dot{\kappa}_{i}=\lambda f a l\left(-L(t) \operatorname{sign}\left(\varpi_{i}\right)-\kappa_{i}, \eta_{i}, \vartheta_{i 0}\right) \\
& \operatorname{fal}\left(X, \eta, \vartheta_{0}\right)=\left\{\begin{array}{l}
|X|^{\eta} \operatorname{sign}(X),|X|>\vartheta_{0} \\
\frac{X}{\vartheta_{0}{ }^{1-\eta}},|X| \leq \vartheta_{0}
\end{array}\right.
\end{aligned}
$$


where $\rho_{i 0}, \lambda, \beta_{i 1}>0,0<\ell_{i 0}, \ell_{i 1}, \eta_{i}, \vartheta_{i 0}<1$ are control parameters. The approximation value of $L_{i} \operatorname{sign}\left(\varpi_{i}\right)$ is obtained by taking a $f a l(\cdot)$ function filter.

\subsection{Fixed-time sliding mode controller}

Based on Eq. (8), the state space expression of the dynamic model of MRMs is:

$$
\left\{\begin{aligned}
\dot{x}_{i 1}= & x_{i 2} \\
\dot{x}_{i 2}= & M_{i} \tau_{i}-M_{i}\left(\hat{B}_{i} \dot{q}_{i}-\left(\hat{F}_{c i}+\hat{F}_{s i} \exp \left(-\hat{F}_{\tau i} \dot{q}_{i}^{2}\right)\right) \operatorname{sign}\left(\dot{q}_{i}\right)\right) \\
& \quad F_{Q}\left(q_{i}, \dot{q}_{i}\right)+M_{i} Y\left(\dot{q}_{i}\right) \tilde{\theta}_{i}-M_{i} \frac{\tau_{f i}}{\gamma_{i}}+\hat{v}_{i}+\varpi_{i}
\end{aligned}\right.
$$

where $\left[x_{i 1}, x_{i 2}\right]=\left[q_{i}, \dot{q}_{i}\right]^{T}$, and $\varpi_{i}$ is the disturbance estimation error and $\varpi_{i}$ is bounded by $\varpi_{i} \leq \Gamma_{i}, \Gamma_{i}$ is a positive constant.

Let $e_{i}=x_{i 1}-x_{i d}, r_{i}=\dot{e}_{i}+\lambda_{i} e_{i}, x_{i d}$ is the desired trajectory, and $\lambda_{i}$ is a positive constant. Then, to achieve a fixed-time control, fixed-time sliding mode surface [19] is designed as

$$
s_{i}=\dot{e}_{i}+\alpha_{i} \llbracket e_{i} \rrbracket^{\mu_{i}}+\beta_{i} \llbracket e_{i} \rrbracket^{\nu_{i}}
$$

where $0<\nu_{i}<1$ and $\mu_{i}>1$. The gain $\alpha_{i}, \beta_{i}$ are positive constants.

Differentiating (21) results in

$$
\dot{s}_{i}=\ddot{e}_{i}+\alpha_{i} \mu_{i}\left|e_{i}\right|^{\mu_{i}-1} \dot{e}_{i}+\beta_{i} \nu_{i}\left|e_{i}\right|^{\nu_{i}-1} \dot{e}_{i}
$$

For 22), If $e_{i}=0$ and $\dot{e}_{i} \neq 0$, there will be singularity problems because of $\alpha_{i}-1<0$. Inspired by the above sliding mode hyperplane, a novel type of fixed-time SMC hyperplane is designed, the expression is as follows:

$$
s_{i}=e_{i}+c_{i}^{-1 / m_{i}} \llbracket \dot{e}_{i}+b_{i} \llbracket e_{i} \rrbracket^{n_{i}} \rrbracket^{\frac{1}{m_{i}}}
$$

where $c_{i}>0, b_{i}>0, \frac{1}{2}<m_{i 1}<1$ and $n_{i}>1$.

Remark 2: when $s_{i}=0,23$ implies $\dot{e}_{i}=-b_{i} \llbracket e_{i} \rrbracket^{n_{i}}-c_{i} \llbracket e_{i} \rrbracket^{m_{i}}$, which can be verified based on Lemma 1 that the settling time $T_{s}$ is bounded by $T_{s}<T_{\max } \triangleq$ ${ }_{140} \frac{1}{b_{i}\left(n_{i}-1\right)}+\frac{1}{c_{i}\left(1-m_{i}\right)}$ 


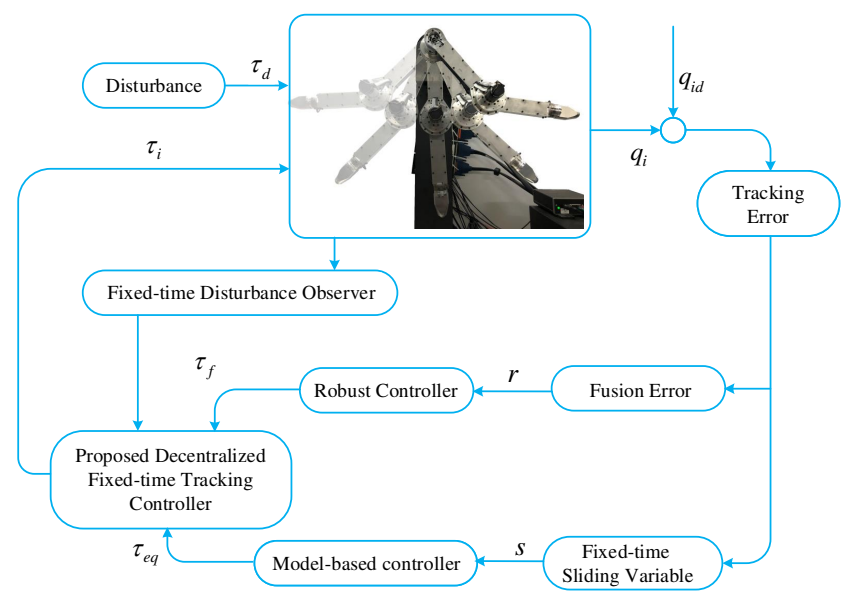

Figure 2: Schematic diagram of the presented control method.

The derivative of Eq. 23 with respect to time is given:

$$
\dot{s}_{i}=\dot{e}_{i}+c_{i}{ }^{-\frac{1}{m_{i}}} \frac{1}{m_{i}}\left|\dot{e}_{i}+b_{i} \llbracket e_{i} \rrbracket^{n_{i}}\right|^{\frac{1}{m_{i}}-1}\left(\ddot{e}_{i}+b_{i} n_{i}\left|e_{i}\right|^{n_{i}-1} \dot{e}_{i}\right)
$$

Inserting (20) into $(24)$ yields

$$
\begin{aligned}
& \dot{s}_{i}=\dot{e}_{i}+c_{i}{ }^{-\frac{1}{m_{i}}} \frac{1}{m_{i}}\left|\dot{e}_{i}+b_{i} \llbracket e_{i} \rrbracket^{n_{i}}\right|^{\frac{1}{m_{i}}-1}\left(\dot{x}_{i 2}-\ddot{x}_{i d}+b_{i} n_{i}\left|e_{i}\right|^{n_{i}-1} \dot{e}_{i}\right) \\
&=\dot{e}_{i}+c_{i}{ }^{-\frac{1}{m_{i}}} \frac{1}{m_{i}}\left|\dot{e}_{i}+b_{i} \llbracket e_{i} \rrbracket^{n_{i}}\right|^{\frac{1}{m_{i}}-1}\left(\begin{array}{l}
M_{i} \tau_{i}+F_{Q}\left(q_{i}, \dot{q}_{i}\right)-M_{i} \frac{\tau_{f i}}{\gamma_{i}}+M_{i} Y\left(\dot{q}_{i}\right) \widetilde{\theta}_{i}+\hat{v}_{i} \\
-M_{i}\left(\hat{B}_{i} \dot{q}_{i}+\left(\hat{F}_{c i}+\hat{F}_{s i} \exp \left(-\hat{F}_{\tau i} \dot{q}_{i}^{2}\right)\right) \operatorname{sign}\left(\dot{q}_{i}\right)\right) \\
+\varpi_{i}-\ddot{x}_{i d}+b_{i} n_{i}\left|e_{i}\right|^{n_{i}-1} \dot{e}_{i}
\end{array}\right)
\end{aligned}
$$

The decentralized control law is designed as:

$$
\tau_{i}=\tau_{f}+\tau_{e q}
$$

Design compensator $\tau_{f}$ to compensate non-parametric friction terms $F_{Q}\left(q_{i}, \dot{q}_{i}\right)$, the specific expression form is as follows:

$$
\tau_{f}=\left\{\begin{array}{cc}
-\rho_{i} \frac{r_{i}}{\left|r_{i}\right|} & \left|r_{i}\right|>\varepsilon_{i} \\
-\rho_{i} \frac{r_{i}}{\varepsilon_{i}} & \left|r_{i}\right| \leq \varepsilon_{i}
\end{array}\right.
$$

where $\varepsilon_{i}$ is a tunable positive constant. 
If the parameter uncertainty can be taken into account as an unknown constant, it can be compensated by an integral compensator. However, in practical engineering applications, the parameter uncertainty of the model varies with factors such as temperature, and $\tilde{\theta}_{i}$ is decomposed as:

$$
\tilde{\theta}_{i}=\tilde{\theta}_{c}+\tilde{\theta}_{v}
$$

where, $\tilde{\theta}_{c}$ is a constant unknown vector, and $\tilde{\theta}_{v}$ is a variable with an upper limit, i.e, $\left|\tilde{\theta}_{v}^{i}\right|<\rho_{n}^{i}, n=1,2,3,4,5,6$. In order to compensate $\tilde{\theta}_{c}$ and $\tilde{\theta}_{v}$, the following compensation controller is designed:

$$
\begin{gathered}
u_{p c}^{i}=-k_{1} \int_{0}^{t} Y\left(\dot{q}_{i}\right)^{T} r_{i} d \tau \\
u_{p v n}^{i}=\left\{\begin{array}{l}
-\rho_{n}^{i} \frac{\varsigma_{n}^{i}}{\left|\varsigma_{n}^{i}\right|},\left|\varsigma_{n}^{i}\right|>\varepsilon_{p n}^{i} \quad n=1,2,3,4,5,6 \\
-\rho_{n}^{i} \frac{\varsigma_{n}^{i}}{\varepsilon_{p n}^{i}},\left|\varsigma_{n}^{i}\right| \leq \varepsilon_{p n}^{i}
\end{array}\right.
\end{gathered}
$$

where $\varsigma_{n}^{i}=Y\left(\dot{q}_{i}\right)^{T} r_{i}$, and $\varepsilon_{p n}^{i}$ is the positive control parameter.

$$
\begin{aligned}
\tau_{e q}= & M_{i}^{-1}\left(\begin{array}{r}
\ddot{x}_{i d}-\hat{v}_{i}-b_{i} n_{i}\left|e_{i}\right|^{n_{i}-1} \dot{e}_{i}-c_{i}^{\frac{1}{m_{i}}} m_{i}\left|\dot{e}_{i}+b_{i} \llbracket e_{i} \rrbracket^{n_{i}}\right|^{2-\frac{1}{m_{i}}} \dot{e}_{i} \\
-\left(\Gamma_{i}+a_{i}\right) \operatorname{sign}\left(s_{i}\right)-\lambda_{i 1} \llbracket s_{i} \rrbracket^{m_{i 1}}-\lambda_{i 2} \llbracket s_{i} \rrbracket^{m_{i 2}}
\end{array}\right) \\
& +\hat{B}_{i} \dot{q}_{i}+\left(\hat{F}_{c i}+\hat{F}_{s i} \exp \left(-\hat{F}_{\tau i} \dot{q}_{i}^{2}\right)\right) \operatorname{sign}\left(\dot{q}_{i}\right)+Y\left(\dot{q}_{i}\right)\left(u_{p c}^{i}+u_{p v}^{i}\right)+\frac{\tau_{f i}}{\gamma_{i}}
\end{aligned}
$$

Theorem 2. Considering the MRMs system (20), the decentralized fixedtime tracking controller is designed as (26). The system error of MRMs will converge to zero within fixed-time, and under Lemma 1, the settling time is bounded by:

$$
T<T_{\max } \triangleq T_{0}+T_{1}+T_{s}+\varepsilon(\tau)
$$

where $T_{0}, T_{1}$ and $T_{s}$ are defined in (17), Remark 2, and (36). $\varepsilon(\tau)$ is a small time margin related to the boundary width.

The following Lyapunov candidate and its time derivative are computed as: 


$$
\begin{aligned}
& V_{i 2}=0.5 \sum_{i=1}^{n} s_{i}^{2} \Rightarrow \dot{V}_{i 2}=\sum_{i=1}^{n} s_{i} \dot{s}_{i} \\
& =\sum_{i=1}^{n} s_{i}\left(\dot{e}_{i}+\Delta_{i}\left(\begin{array}{l}
\left.M_{i} \tau_{i}-M_{i}\left(\hat{B}_{i} \dot{q}_{i}-\left(\hat{F}_{c i}+\hat{F}_{s i} \exp \left(-\hat{F}_{\tau i} \dot{q}_{i}^{2}\right)\right) \operatorname{sign}\left(\dot{q}_{i}\right)\right)\right) \\
+F_{Q}\left(q_{i}, \dot{q}_{i}\right)-M_{i} \frac{\tau_{f i}}{\gamma_{i}}+\hat{v}_{i}+\varpi_{i}-\ddot{x}_{i d}+b_{i} n_{i}\left|e_{i}\right|^{n_{i}-1} \dot{e}_{i}
\end{array}\right)\right) \\
& \left.=\sum_{i=1}^{n} s_{i}\left(\begin{array}{l}
\ddot{x}_{i d}-\hat{v}_{i}-b_{i} n_{i}\left|e_{i}\right|^{n_{i}-1} \dot{e}_{i}-c_{i} \frac{1}{m_{i}} m_{i}\left|\dot{e}_{i}+b_{i} \llbracket e_{i} \rrbracket^{n_{i}}\right|^{2-\frac{1}{m_{i}}} \dot{e}_{i} \\
-\left(\Gamma_{i}+a_{i}\right) \operatorname{sign}\left(s_{i}\right)-\lambda_{i 1} \llbracket s_{i} \rrbracket^{m_{i 1}}-\lambda_{i 2} \llbracket s_{i} \rrbracket^{m_{i 2}}+M_{i} \frac{\tau_{f i}}{\gamma_{i}} \\
+M_{i}\left(\hat{B}_{i} \dot{q}_{i}+\left(\hat{F}_{c i}+\hat{F}_{s i} \exp \left(-\hat{F}_{\tau i} \dot{q}_{i}^{2}\right)\right) \operatorname{sign}\left(\dot{q}_{i}\right)\right) \\
+M_{i} \tau_{f}-M_{i}\left(\hat{B}_{i} \dot{q}_{i}-\left(\hat{F}_{c i}+\hat{F}_{s i} \exp \left(-\hat{F}_{\tau i} \dot{q}_{i}^{2}\right)\right) \operatorname{sign}\left(\dot{q}_{i}\right)\right) \\
-F_{Q}\left(q_{i}, \dot{q}_{i}\right)-M_{i} \frac{\tau_{f i}}{\gamma_{i}}+\hat{v}_{i}+\varpi_{i}-\ddot{x}_{i d}+b_{i} n_{i}\left|e_{i}\right|^{n_{i}-1} \dot{e}_{i}
\end{array}\right)\right)
\end{aligned}
$$

For $i$ th joint, and $\mathrm{n}=1,2,3,4,5,6$, if $\left|\varsigma_{n}^{i}\right|>\varepsilon_{p n}^{i}$, exist $\varsigma_{n}^{i}\left(\tilde{\theta}_{v}+u_{p v n}^{i}\right)<0$, if $\left|\varsigma_{n}^{i}\right| \leq \varepsilon_{p n}^{i}, \varsigma_{n}^{i}\left(\tilde{\theta}_{v}+u_{p v n}^{i}\right) \leq \varsigma_{n}^{i} \sum_{n=1}^{6}\left(\rho_{n}^{i} \frac{\varsigma_{n}^{i}}{\left|\varsigma_{n}^{i}\right|}-\rho_{n}^{i} \frac{\varsigma_{n}^{i}}{\varepsilon_{p n}^{i}}\right)$, and reaches its maximum at the $\left|\varsigma_{n}^{i}\right| \leq \frac{\varepsilon_{p n}^{i}}{2}$.

$$
\begin{aligned}
\dot{V}_{i 2} & \leq \Delta_{i}\left(-\lambda_{i 1} \sum_{i=1}^{n}\left|s_{i}\right|^{m_{i 1}+1}-\lambda_{i 2} \sum_{i=1}^{n}\left|s_{i}\right|^{m_{i 2}+1}+\sum_{n=1}^{6} \frac{\rho_{n}^{i} \varepsilon_{p n}^{i}}{4}\right) \\
& \leq \Delta_{i}\left(-2^{\frac{m_{i 1}+1}{2}} 2^{\frac{m_{i 1}}{2}} \lambda_{i 1}\left(V_{i 2}^{\frac{m_{i 1}+1}{2}}\right)-2^{\frac{m_{i 2}+1}{2}} \lambda_{i 2}\left(V_{i 2}^{\frac{m_{i 2}+1}{2}}\right)+\ell\right)
\end{aligned}
$$

where $\Delta_{i}=c_{i}^{-\frac{1}{m_{i}}} \frac{1}{m_{i}}\left|\partial_{i}\right|^{\frac{1}{m_{i}}-1}, \partial_{i}=\dot{e}_{i}+b_{i} \llbracket e_{i} \rrbracket^{n_{i}}, \ell=\sum_{n=1}^{6} \frac{\rho_{n}^{i} \varepsilon_{p n}^{i}}{4}$. In order

to facilitate the analysis, the state $\left(e_{i}, \dot{e}_{i}\right)$ is divided into two regions $\Omega_{i 1}=$ $\left\{\left(e_{i}, \dot{e}_{i}\right) \mid \Delta_{i} \geq 1\right\}$ and $\Omega_{i 2}=\left\{\left(e_{i}, \dot{e}_{i}\right) \mid \Delta_{i}<1\right\}$.

i. If the system state is in area $\Omega_{i 1}$, formula (37) can be obtained:

$$
\dot{V}_{i 2} \leq-2^{\frac{m_{i 1}+1}{2}} 2^{\frac{m_{i 1}}{2}} \lambda_{i 1}\left(V_{i 2}^{\frac{m_{i 1}+1}{2}}\right)-2^{\frac{m_{i 2}+1}{2}} \lambda_{i 2}\left(V_{i 2}^{\frac{m_{i 2}+1}{2}}\right)+\ell
$$

It is worth noting that $V_{i}=0$ can be understood as $s_{i}=0$. This means that the system is stable at a fixed-time and has a bounded settling time $T_{s}$, and is

$$
T_{s} \leq \frac{1}{2^{\frac{m_{i 1}+1}{2}} n^{\frac{1-m_{i 1}}{2}} \lambda_{i 1}\left(\frac{m_{i 1}+1}{2}-1\right) \ell}+\frac{1}{2^{\frac{m_{i 2}+1}{2}} \lambda_{i 2}\left(1-\frac{m_{i 2}+1}{2}\right) \ell}
$$


ii. If the system state is in area $\Omega_{i 2}, 0<\Delta_{i}<1$, when $\partial_{i} \neq 0$. The terminal sliding mode hyperplane $s_{i}=0$ can still prove to be an attractor. What remains is to prove that $\partial_{i}=0$ is not attractive except for the origin [40]. Therefore, it can be concluded that the sliding surface $s=0$ can be reached from anywhere in the phase plane within fixed time $t_{r}<T_{r}+\varepsilon(\tau)$ [40. Once the sliding surface $s=0$ is reached, it follows from (21), the system states will reach the origin within the fixed time $T_{s}$. Hence, the total settling time $T$ is bounded by 32 . This completes the proof.

Remark 3: Although fixed-time control has attracted widespread attention, the global fixed-time stability of the robot system remains to be studied. This paper proposes a novel decentralized fixed-time control, which achieves fixedtime convergence, strong robustness, and high-precision tracking control effects. The main contributions of this paper are: 1) The extended state based fixed time observer is designed to compensate for the influence of uncertain terms in the controller, and the adaptive value is obtained through the principle of the twolayer adaptive. The adaptive value adapts according to the change of the total uncertainty, avoiding the need for the upper bound of uncertainty. 2) Compared with TSMC, a novel decentralized fixed-time sliding mode control proposed in this paper has higher accuracy, faster convergence speed, and effectively reduces chattering.

\section{Theoretical and experimental analysis}

\subsection{Simulations}

Considering the tracking problem for MRMs with model uncertainties and uncertain disturbances, the validity of fixed-time controller is verified in this subsection through numerical simulations. The parameter settings of MRMs are designed in Table 1, including the nominal parameters of the friction model, numerical simulation parameters, and experimental parameters. 
Table 1: Parameters setting

\begin{tabular}{|c|c|c|c|c|c|c|}
\hline Parameter type & Name & Value & Name & Value & Name & Value \\
\hline \multirow[t]{2}{*}{ Model parameters } & $\hat{B}_{i}$ & $0.0123 \mathrm{Nms} / \mathrm{rad}$ & $\hat{F}_{\tau i}$ & $20 s^{2} / r a d^{2}$ & $I_{m i}$ & $118 \mathrm{gcm}^{2}$ \\
\hline & $\hat{F}_{c i}$ & $0.0015 \mathrm{Nm}$ & $\hat{F}_{s i}$ & $0.02557 \mathrm{Nm}$ & $\gamma_{i}$ & 101 \\
\hline Numerical simulation & $\alpha_{i} \beta_{i}$ & 58 & $\varphi_{i} \psi_{i}$ & $1.5 \quad 0.6$ & $\varsigma_{i} \xi_{i}$ & 73 \\
\hline \multirow[t]{4}{*}{ control parameters } & $\mu_{i} \quad \nu_{i}$ & $1.25 \quad 0.5$ & $m_{i} n_{i}$ & $0.75 \quad 5 / 3$ & $c_{i} \quad b_{i}$ & 1020 \\
\hline & $\Gamma_{i} a_{i}$ & $45 \quad 1.5$ & $\lambda_{i 1} \quad \lambda_{i 2}$ & 1515 & $m_{i 1} \quad m_{i 2}$ & $\begin{array}{ll}1.75 & 0.75\end{array}$ \\
\hline & $\varepsilon_{i} \rho_{i}$ & $0.1 \quad 0.07$ & $\lambda_{i} \vartheta_{0}$ & $100 \quad 0.6$ & $\rho_{i 0} \quad \beta_{i 1}$ & 23 \\
\hline & $\ell_{i 1} \quad \ell_{i 0}$ & $1.2 \quad 0.75$ & $\lambda \eta$ & 40.6 & & \\
\hline \multirow[t]{5}{*}{ Experimental control parameters } & $\lambda_{i 1} \quad \lambda_{i 2}$ & $17 \quad 13$ & $\varepsilon_{i} \quad \rho_{i}$ & $0.1 \quad 0.07$ & $\begin{array}{ll}\lambda_{i} & \vartheta_{0}\end{array}$ & 1202 \\
\hline & $\alpha_{i} \beta_{i}$ & 1012 & $\varphi_{i} \psi_{i}$ & $1.75 \quad 0.75$ & $\varsigma_{i} \xi_{i}$ & 125 \\
\hline & $\mu_{i} \quad \nu_{i}$ & $1.75 \quad 0.75$ & $m_{i} n_{i}$ & $0.75 \quad 9 / 7$ & $c_{i} \quad b_{i}$ & 1325 \\
\hline & $\Gamma_{i} a_{i}$ & $\begin{array}{ll}50 & 1.5\end{array}$ & $m_{i 1} \quad m_{i 2}$ & $1.75 \quad 0.55$ & $\rho_{i 0} \quad \beta_{i 1}$ & 35 \\
\hline & $\ell_{i 1} \quad \ell_{i 0}$ & $2 \quad 0.75$ & $\lambda \eta$ & $\begin{array}{ll}5 & 0.6\end{array}$ & & \\
\hline
\end{tabular}

The tracking trajectory of the two joints is as follows

$$
\begin{array}{ll}
q_{1 d}=0.5 \sin (2.2 t)-0.3 \sin (1.5 t), & 0 \leq t \leq 20 \\
q_{2 d}=0.5 \sin (0.6 t)-0.3(\cos (2 t)-1), & 0 \leq t \leq 20
\end{array}
$$

Case 1: The initial positions are $\left[q_{1}(0), q_{2}(0)\right]^{T}=[0.04,0.02]^{T}$. The position tracking error comparison of the numerical simulation is shown in Fig 3 and Fig 4 . The above figures show that the system produces a large error fluctuation during the initial operation and quickly returns to the equilibrium point. After stabilization, the residuals near the equilibrium point are small, which proves that the system error reaches the equilibrium point in a fixed time. From the simulation results that the proposed control approach achieves a good tracking process and has higher control accuracy under model uncertainty and uncertain disturbance. Fig 5 and Fig 6 show the control torque of two different control methods under joint 1 and joint 2 . As shown in the figures, since the proposed control strategy estimates the total uncertainty through the observer, the chat- 
tering phenomenon has been significantly weakened. In addition, the proposed fixed-time control law also helps to reduce chattering.

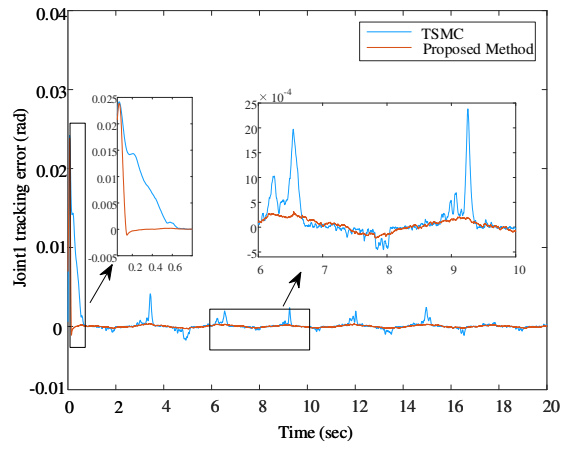

Figure 3: Joint 1 tracking error curves

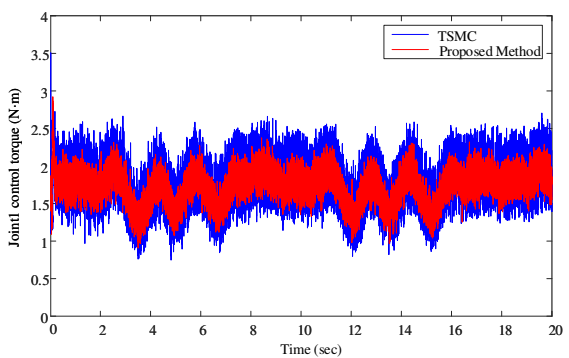

Figure 5: Joint 1 control torque curves

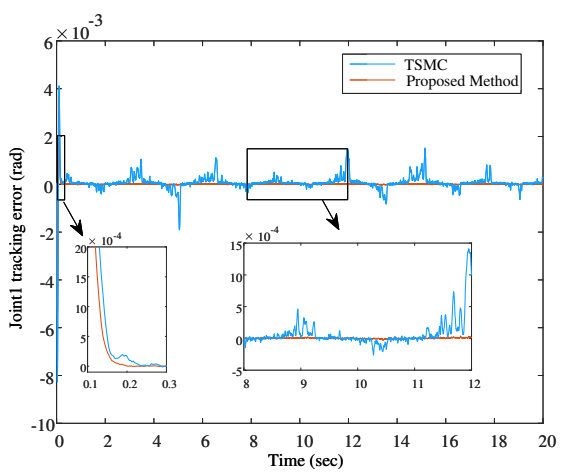

Figure 7: Joint 1 tracking error curves

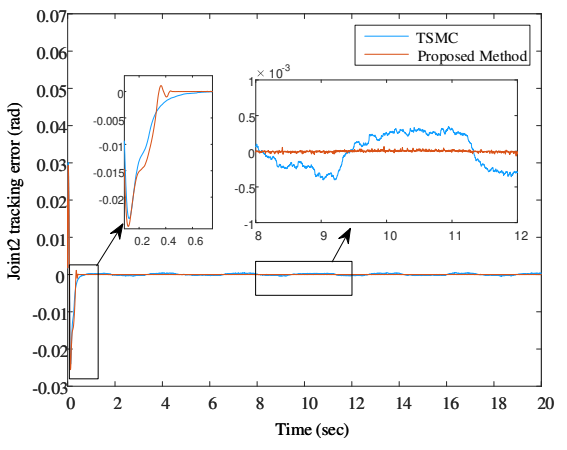

Figure 4: Joint 2 tracking error curves

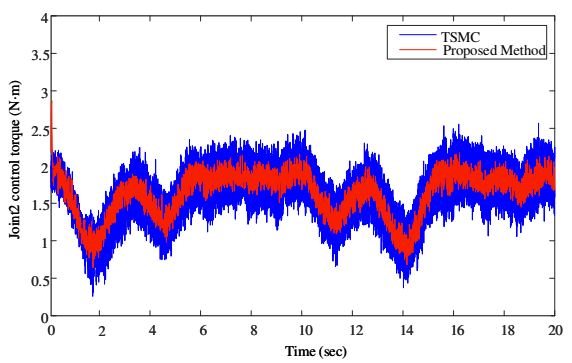

Figure 6: Joint 2 control torque curves

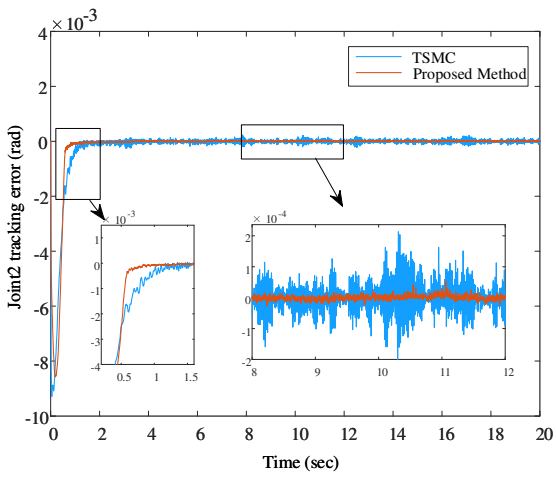

Figure 8: Joint 2 tracking error curves 


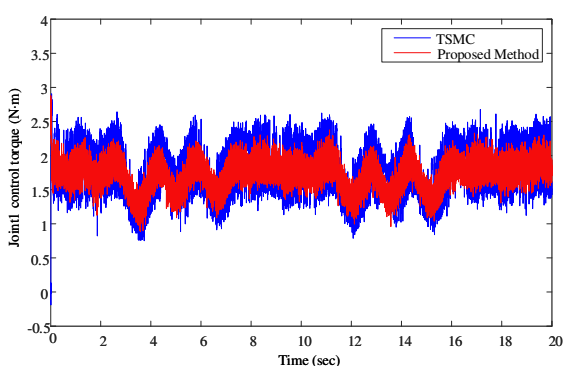

Figure 9: Joint 1 control torque curves

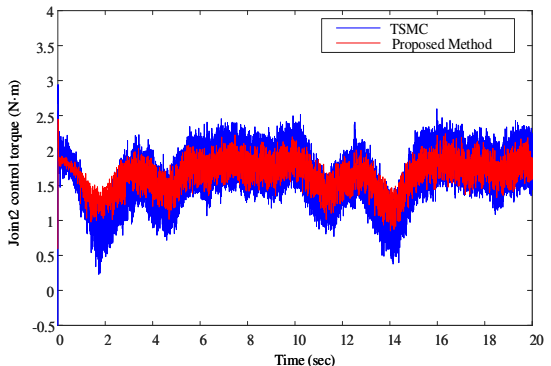

Figure 10: Joint 2 control torque curves

Case 2: The initial conditions are zero. The position tracking error comparison of the numerical simulation is shown in Fig 7 and Fig 8 Fig 9 and Fig 10 show the control torque of two different control methods under joint 1 and joint 2. The two control methods compared provide high tracking accuracy and fast convergence. Therefore, they can be utilized for trajectory tracking control of uncertain MRM. Note that the numerical simulation parameters are the same in both cases, only the initial conditions are different.

\subsection{Experimental analysis}

The proposed decentralized fixed-time tracking control approach is further verified on a two-link MRMs experimental platform, as shown in Fig 11. The two-link MRMs consist of two sets of joint modules and links. Each joint module contains a DC brushed motor produced by Maxon, with a maximum output torque of $190 \mathrm{mNm}$. A harmonic drive device is connected to the output of the motor, and an incremental encoder produced by Maxon is installed on the motor end with an accuracy of 500 count/rev to measure the displacement of the motor end. An absolute encoder produced by Netzer is installed on the link end, which has a 19-bit precision and is used to collect the absolute position data of the link end. Adopt the advanced power amplifier (PLA) produced by Quanser Company to drive the motor of the joint module and the QPIDe data acquisition card to collect the data of each sensor. The joint torque sensor is installed between the link module and the joint module to measure the joint 
coupling torque.

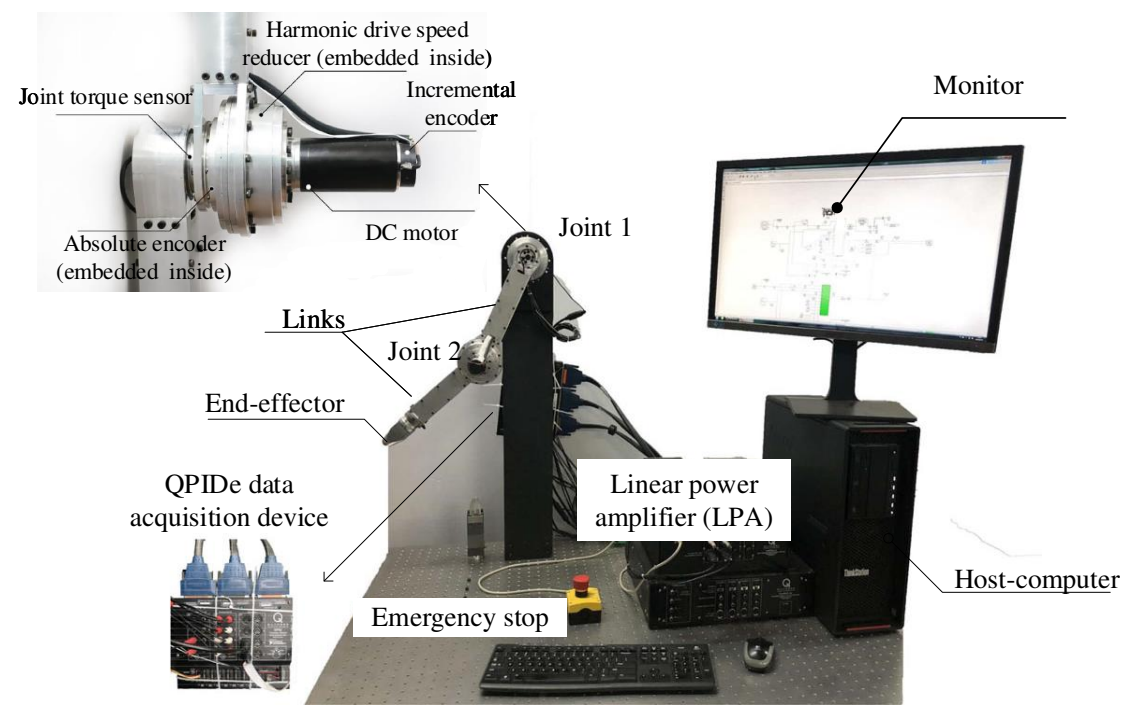

Figure 11: Experimental platform.

An experiment of two-link MRMs with tracking control is built to validate the effectiveness of the proposed control algorithm. Numerical simulations and experiments show that the uncertain disturbance conditions are the same, $\tau_{1 d}=$ $0.02 q_{1 d}^{2}$ and $\tau_{2 d}=0.02 s i n q_{2 d}$ for joint 1 and joint 2 , respectively. The parameters of TSMC are $\beta_{i}=4, q_{i}=5$, and $p_{i}=3$ [17]. All the initial conditions are zero. The desired positions are set as:

$$
\begin{array}{ll}
q_{1 d}=0.2 \sin (3 \pi t / 10), & 0 \leq t \leq 20 \\
q_{2 d}=0.2 \sin (\pi t / 5), & 0 \leq t \leq 20
\end{array}
$$

Figs 12 and 13 show the tracking curves of MRMs under the TSMC and the proposed decentralized fixed-time control strategy, respectively. From these figures, both methods ensure good tracking performance of the MRMs system, which signifies that the trajectory tracking task is successfully achieved under the two control methods. The trajectory error of the system is shown in Figs. 14, 15. and the two control methods keep the tracking error of each joint within ${ }_{215}$ an acceptable range. From the error comparison curves, MRMs based on the 
TSMC method has tracking error less than 6e-3 rad and 1.5e-3, respectively. The errors of the proposed decentralized fixed-time control method are less than 4e-3 rad and 0.5e-3 rad, respectively. From the control torque curves in Figs. 16, 17. it can be seen that the proposed control method has a certain effect on reducing chattering. As we can see, the two control methods ensure good tracking performance. Similar to the numerical simulation, the proposed decentralized fixed-time control approach is better than the performance of TSMC.

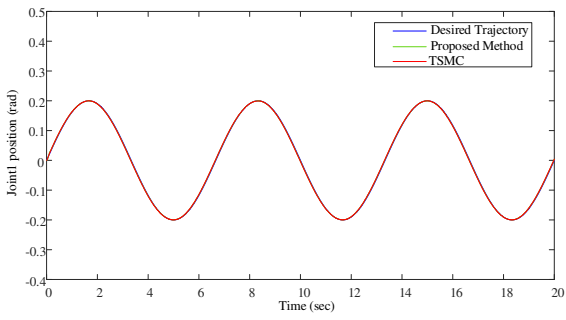

Figure 12: Joint 1 position curves.

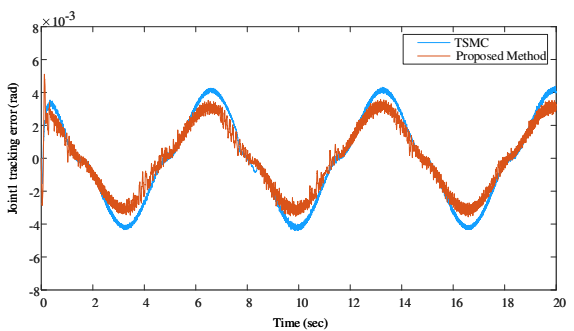

Figure 14: Joint 1 error curves.

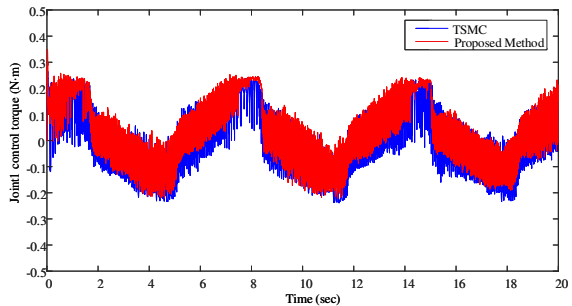

Figure 16: Joint 1 control torque curves.

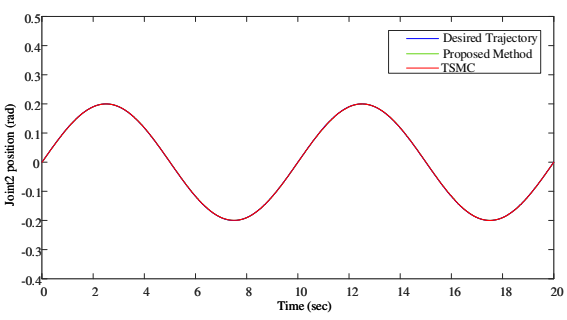

Figure 13: Joint 2 position curves.

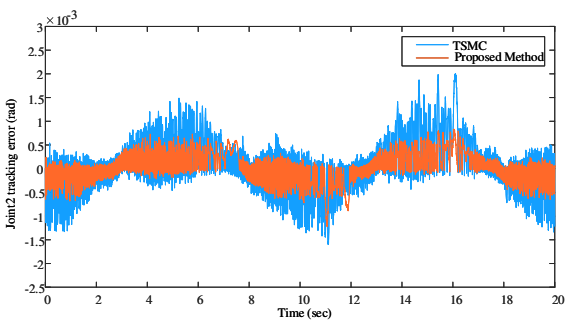

Figure 15: Joint 2 error curves.

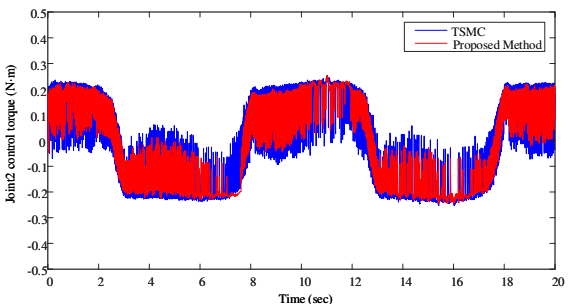

Figure 17: Joint 2 control torque curves. 


\section{Conclusion}

This paper uses a fixed-time control algorithm based on the extended state disturbance observer to solve model uncertainties and uncertain disturbances of MRMs and the decentralized tracking control problem of uncertain disturbance. With the help of JTF technology, a dynamic model of MRMs subsystem is established. The saturation-based robust controller is used to avoid the influence of non-parametric friction terms. A novel fixed-time SMC method based on the 230 extended state fixed-time disturbance observer is designed, which is used for the global decentralized fixed-time tracking control of MRMs with model uncertainties and uncertain disturbances. Through the Lyapunov stability theorem, it is demonstrated that the tracking error of MRMs converges in a fixed time under the proposed control method, and the settling time can be set arbitrarily with the design parameters, independent of the initial state of the system. Numerical simulations and experimental results demonstrate that the proposed approach improves the control performance of the system. Future efforts will focus on global fixed-time stable fault-tolerant control for MRMs subject to uncertain environmental disturbances and actuator constraints.

\section{Acknowledgments}

This work is supported by the National Natural Science Foundation of China (Grant no. 61773075 and 61703055), the Scientific Technological Development Plan Project in Jilin Province of China (Grant nos. 20200801056GH and 20200404208YY), and the Science and Technology project of Jilin Provincial Education Department of China during the 13th Five-Year Plan Period (JJKH20200672KJ, JJKH20200673KJ, and JJKH20210767KJ)

\section{Declarations}

\section{Conflict of interest}

The authors declare that they have no conflict of interest. 


\section{Data Availability Statements}

All data generated or analyzed during this study are included in this published article. The model and parameter data are obtained in the table of "parameter setting" in the paper.

\section{References}

[1] A. Yun, D. Moon, J. Ha, S. Kang, W. Lee, Modman: an advanced reconfigurable manipulator system with genderless connector and automatic kinematic modeling algorithm, IEEE Robotics and Automation Letters $5(3)(2020)$, pp. 4225-4232.

[2] H.D. Yang, A.T. Asbeck,Design and characterization of a modular hybrid continuum robotic manipulator, IEEE/ASME Transactions on Mechatronics 25(6)(2020),pp. 2812-2823.

[3] Q.X. Wu, X.K. Wang, L. Hua, M.H. Xia, Modeling and nonlinear sliding mode controls of double pendulum cranes considering distributed mass beams, varying roped length and external disturbances, Mechanical Systems and Signal Processing 158 (2021).

[4] R. Hu, H. Deng, Y. Zhang, Novel dynamic-sliding-mode-manifold-based continuous fractional-order nonsingular terminal sliding mode control for a class of second-order nonlinear systems, IEEE Access 8(2020), pp.1982019829 .

[5] H. Zhu, J. Shen, K.Y. Lee, L. Sun, Multi-model based predictive sliding mode control for bed temperature regulation in circulating fluidized bed boiler, Control Engineering Practice, 101(2020), pp.104484.

[6] Z. Wang, Y. Liu, Z. Guan, Y. Zhang, An adaptive sliding mode motion control method of remote operated vehicle, IEEE Access 9 (2021), pp.2244722454 . 
[7] V.T. Do, S.G. Lee, J.H. Kim, Robust integral backstepping hierarchical sliding mode controller for a ballbot system. Mechanical Systems and Signal Processing 144 (2020):106866.

[8] K. Rsetam, Z. Cao, Z. Man, Cascaded-extended-state-observer-based sliding-mode control for underactuated flexible joint robot, IEEE Transactions on Industrial Electronics 67(12) (2020) 10822-10832.

[9] S Yu. X. Yu. B. Shirinzadeh. Z. Man. Continuous finite-time control for robotic manipulators with terminal sliding mode, Automatica, 4(11)(2005), pp. 1957-1964.

[10] L. Ren, G. Lin, Y. Zhao, Z. Liao, F. Peng, Adaptive nonsingular finitetime terminal sliding mode control for synchronous reluctance motor, IEEE Access 9(2021), pp. 51283-51293.

[11] Y. Chu, S. Hou, J Fei, Continuous terminal sliding mode control using novel fuzzy neural network for active power filter, Control Engineering Practice,109(4)(2021), pp.04735.

[12] J. Pliego-Jiménez, M.A. Arteaga-Pérez, M. López-Rodríguez. Finite-time control for rigid robots with bounded input torques, Control Engineering Practice, 102(1)(2020), pp.104556.

[13] Y.J Wu, G.F. Li, Adaptive disturbance compensation finite control set optimal control for PMSM systems based on sliding mode extended state observer, Mech. Syst. Sig. Process. 98 (2018) 402-414.

[14] Ruchika, N. Kumar, Finite time control scheme for robot manipulators using fast terminal sliding mode control and RBFNN, International Journal of Dynamics \& Control 7(6)(2019) 758-766.

[15] B. Ren, Y. Wang, J. Chen, A novel robust finite-time trajectory control with the high-order sliding mode for human-robot cooperation, IEEE Access 7(2019)130874-130882. 
[16] M. Van, S.S. Ge, H. Ren, Finite time fault tolerant control for robot manipulators using time delay estimation and continuous nonsingular fast terminal sliding mode control, IEEE Transactions on Cybernetics 47(7)(2017) 1681-1693.

[17] Y. Li, Z.P. Lu, F. Zhou, B.Dong, K.P Liu, Y.C. Li, Decentralized trajectory tracking control for modular and reconfigurable robots with torque sensor: adaptive terminal sliding control-based approach, Journal of Dynamic Systems Measurement and Control 141(6)(2019).

[18] G. Zhong, C. Wang, W. Dou, Fuzzy adaptive PID fast terminal sliding mode controller for a redundant manipulator, Mechanical Systems and Signal Processing 159(6)(2021)107577.

[19] D. Cruz-Ortiz, I. Chairez, A. Poznyak, Non-singular terminal sliding-mode control for a manipulator robot using a barrier Lyapunov function, ISA Transactions (2021). https://doi.org/10.1016/j.isatra.2021.04.001.

[20] Z.Y. Zuo, Non-singular fixed-time terminal sliding mode control of nonlinear systems, IET Control Theory Appl. 9(4) (2015) 545-552.

[21] O. Mechali, L. Xu, Y. Huang, M. Shi, X. Xie, Observer-based fixed-time continuous nonsingular terminal sliding mode control of quadrotor aircraft under uncertainties and disturbances for robust trajectory tracking: theory and experiment, Control Engineering Practice, 111(2021), pp. 104806.

[22] S. Song, J. H. Park, B. Zhang, X. Song, Event-triggered adaptive practical fixed-time trajectory tracking control for unmanned surface vehicle, IEEE Transactions on Circuits and Systems II: Express Briefs 68(1)(2021)436440.

[23] L. Cao, B. Xiao, M. Golestani, D. Ran, Faster fixed-time control of flexible spacecraft attitude stabilization, IEEE Transactions on Industrial Informatics 16(2)(2020) 1281-1290. 
[24] Y. Wu, G. Li, Z. Zuo, X. Liu, P. Xu, Practical fixed-time position tracking control of permanent magnet DC torque motor systems, IEEE/ASME Transactions on Mechatronics 26(1)(2021) 563-573.

[25] B. Ning, Q.L. Han, Q. Lu, Fixed-time leader-following consensus for multiple wheeled mobile robots, IEEE Transactions on Cybernetics 50(10) (2020)

[26] L. Zhang, H. Liu, D. Tang, Y. Hou, Y. Wang, Adaptive fixedtime fault-tolerant tracking control and its application for robot manipulators, IEEE Transactions on Industrial Electronics (2021). https://doi.org/10.1109/TIE.2021.3070494.

340 [27] S. Chang, Y. Wang, Z. Zuo, Fixed-time active disturbance rejection control and its application to wheeled mobile robots, IEEE Transactions on Systems, Man, and Cybernetics: Systems (2020)1-11.

[28] Y.X. Su, C.H. Zheng, P. Mercorelli, Robust approximate fixed-time tracking control for uncertain robot manipulators, Mechanical Systems and Signal Processing 135(2020)106379.

[29] C. Hwang, W. Yu, Tracking and cooperative designs of robot manipulators using adaptive fixed-time fault-tolerant constraint control, IEEE Access 8 (2020) 56415-56428.

[30] A. Altan, R. Hacıog, Model predictive control of three-axis gimbal system mounted on UAV for real-time target tracking under external disturbances, Mechanical Systems and Signal Processing 138(2020)106548.

[31] X.L. Shao, N.Liu, Z.Q. Wang, W.D. Zhang, W. Yang, Neuroadaptive integral robust control of visual quadrotor for tracking a moving object, Mechanical Systems and Signal Processing 136(2020)106513.

355

[32] S.K. Kommuri, S.Han, S. Lee, External torque estimation
using higher-order sliding mode observer for robot manip-


ulators, IEEE/ASME Transactions on Mechatronics (2021). https://doi.org/10.1109/TMECH.2021.3067443.

[33] T. Ren, Y. Dong, D. Wu, K. Chen, Collision detection and identification for robot manipulators based on extended state observer, Control Engineering Practice, 79(2018), pp. 144-153.

[34] H. Wang, Z. Zuo, Y. Wang, H. Wang, S. Chang, Composite nonlinear extended state observer and its application to unmanned ground vehicles, Control Engineering Practice, 109(7)(2021), pp.104731.

[35] L. Krzysztof, M. Rafal. Cascade extended state observer for active disturbance rejection control applications under measurement noise, ISA Transactions 109(2021), pp. 1-10.

[36] D. Shi, J. Zhang, Z. Sun, G. Shen, Y. Xia, Composite trajectory tracking control for robot manipulator with active disturbance rejection, Control Engineering Practice, 106(2021), pp. 104670.

[37] G. Liu, S. Abdul, A. Andrew, Distributed control of modular and reconfigurable robot with torque sensing, Robotica 26(1)(2008), pp.75-84.

[38] G. Liu, Decomposition-based friction compensation of mechanical systems, Mechatronics 12(5)(2002)755-769.

375 [39] G. Liu, A.A. Goldenberg, Y. Zhang, Precise slow motion control of a directdrive robot arm with velocity estimation and friction compensation, Mechatronics, 14(7)(2004), pp.821-834.

[40] H. Li, Y. Cai, On SFTSM control with fixed-time convergence, Iet Control Theory \& Applications 11(6)(2017), pp. 766-773.

380 [41] C. Edwards, Y. Shtessel, Adaptive dual-layer super-twisting control and observation, International Journal of Control 89(9)(2016), pp.1759-1766. 


\section{Supplementary Files}

This is a list of supplementary files associated with this preprint. Click to download.

- declarationStatement.pdf 